\title{
Machine log file-based dose verification using novel iterative CBCT reconstruction algorithm in commercial software during volumetric modulated arc therapy for prostate cancer patients
}

\author{
Shoki Inui ${ }^{\text {a,b, }}$, Teiji Nishio ${ }^{\mathrm{b}}$, Yoshihiro Ueda ${ }^{\mathrm{a}}$, Shingo Ohira ${ }^{\mathrm{a}}$, Hikari Ueda ${ }^{\mathrm{a}}$, Hayate Washio ${ }^{\mathrm{a}, \mathrm{c}}$, \\ Shunsuke Ono ${ }^{\mathrm{a}}$, Masayoshi Miyazaki ${ }^{\mathrm{a}}$, Masahiko Koizumi ${ }^{\mathrm{b}}$, Koji Konishi ${ }^{\mathrm{a}}$ \\ ${ }^{a}$ Department of Radiation Oncology, Osaka International Cancer Institute, Osaka, Japan \\ ${ }^{\mathrm{b}}$ Department of Medical Physics and Engineering, Osaka University Graduate School of Medicine, Suita, Japan \\ ${ }^{\mathrm{c}}$ Graduate School of Health Sciences, Kumamoto University, Kumamoto, Japan
}

\section{A R T I C L E I N F O}

\section{Keywords:}

Iterative CBCT

Log file

Prostate cancer

Adaptive radiation therapy

\begin{abstract}
A B S T R A C T
Purpose: To evaluate the utility of the use of iterative cone-beam computed tomography (CBCT) for machine log file-based dose verification during volumetric modulated arc therapy (VMAT) for prostate cancer patients.

Methods: All CBCT acquisition data were used to reconstruct images with the Feldkamp-Davis-Kress algorithm (FDK-CBCT) and the novel iterative algorithm (iCBCT). The Hounsfield unit (HU)-electron density curves for CBCT images were created using the Advanced Electron Density Phantom. The I'mRT and anthropomorphic phantoms were irradiated with VMAT after CBCT registration. Subsequently, fourteen prostate cancer patients received VMAT after CBCT registration. Machine log files and both CBCT images were exported to the PerFRACTION software, and a 3D patient dose was reconstructed. Mean dose for planning target volume (PTV), the bladder, and rectum and the 3D gamma analysis were evaluated.

Results: For the phantom studies, the variation of $\mathrm{HU}$ values was observed at the central position surrounding the bones in FDK-СВCT. There were almost no changes in the difference of doses at the isocenter between measurement and reconstructed dose for planning CT (pCT), FDK-CBCT, and iCBCT. Mean dose differences of PTV, rectum, and bladder between $\mathrm{iCBCT}$ and pCT were approximately $2 \%$ lower than those between FDK-CBCT and pCT. For the clinical study, average gamma analysis for $2 \% / 2 \mathrm{~mm}$ was $98.22 \% \pm 1.07$ and $98.81 \% \pm 1.25 \%$ in FDK-CBCT and iCBCT, respectively.

Conclusions: A similar machine log file-based dose verification accuracy is obtained for FDK-CBCT and iCBCT during VMAT for prostate cancer patients.
\end{abstract}

\section{Introduction}

Recently, high precision radiation therapy, such as volumetric modulated arc therapy (VMAT) and intensity modulated radiation therapy (IMRT), has been performed in many institutes. These techniques can create complicated dose distributions and sharp dose gradients, which increase target conformity and decrease the irradiated volume of organs at risk (OARs). Therefore, patient-specific quality assurance (QA) is necessary, which is mostly performed for pretreatment verification using various measurement devices, such as ion chambers for point dose and radiochromic films or two-dimensional (2D) diode arrays for planar dose distribution [1]. Further, gamma analysis has become a standard method for comparing measured distributions with calculated distributions, using a commercial radiation treatment planning system (TPS) that conforms to AAPM TG-218 report [2]. However, Kuruse et al. [3] reported a lack of correlation between these conventional pre-treatment QA methods and dose errors in anatomic regions of interest. In addition, some previous studies also showed that gamma analysis of planar dose distributions may not directly predict the actual patient dose errors [4-6]. Moreover, pretreatment QA cannot be used to detect inter-fractional variation, such as setup errors and anatomical changes.

To overcome these problems, the method of machine log file-based dose reconstruction has attracted attention as a patient dose volume

\footnotetext{
* Corresponding author at: Department of Radiation Oncology, Osaka International Cancer Institute, 3-1-69 Otemae, Chuou-ku, Osaka 541-8567, Japan.

E-mail address: inui-sy@oici.jp (S. Inui).
} 
(a)
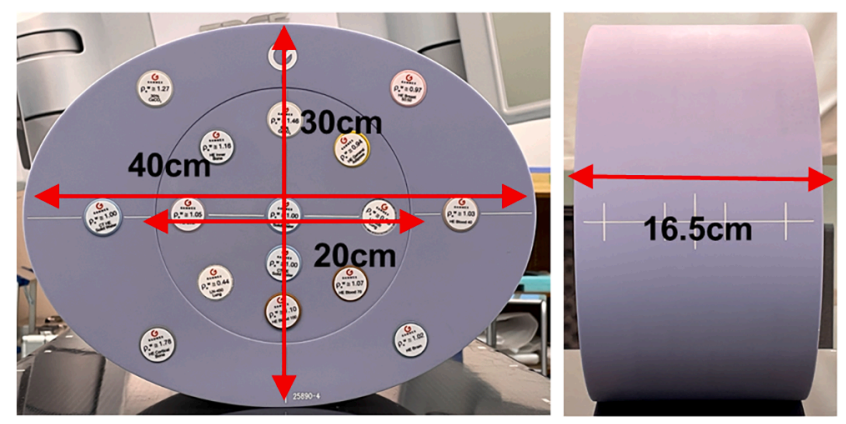

(c)

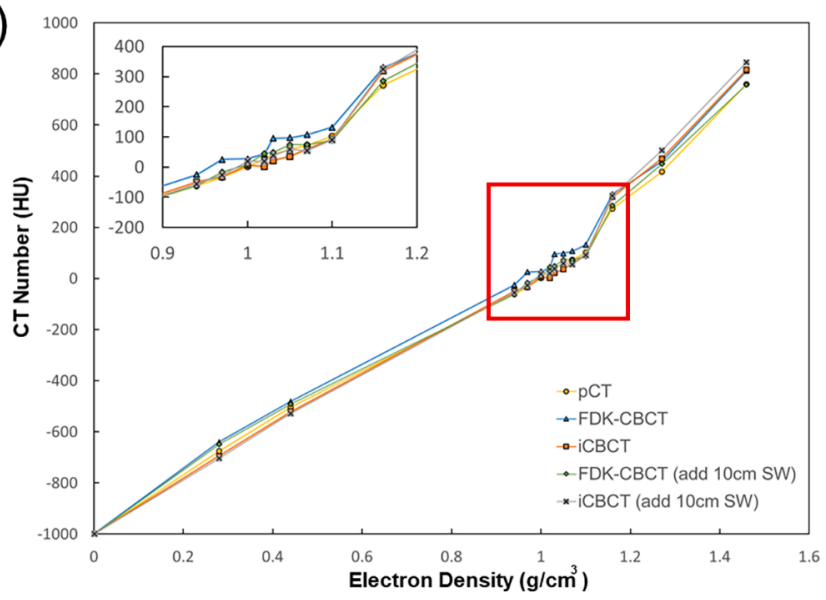

(b)

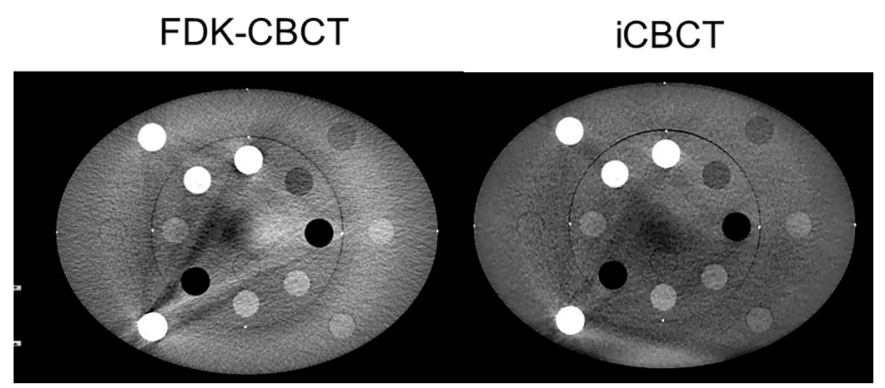

(d)

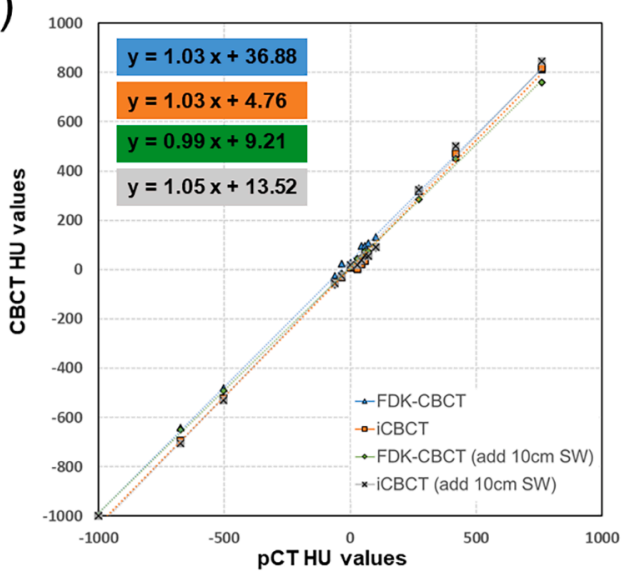

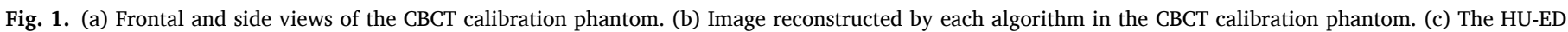
curves for pCT, FDK-CBCT, and iCBCT images simulated with or without $10 \mathrm{~cm}$ SW. (d) Plot of the HU values in pCT and both CBCT images.

histogram (DVH)-based dose verification during IMRT and VMAT. This method uses machine log files, which contain the treatment parameters such as the output of the machine (MU), the gantry angle, and the position of the multi-leaf collimator (MLC). Tyagi et al. [7] showed that machine log file-based dose calculations are in good agreement with actual dose distributions measured using 3D gamma analysis. Accordingly, the use of the machine log file generated by the MLC controller has been proposed as a tool for patient-specific QA during IMRT and VMAT [7-9]. PerFRACTION (Sun Nuclear Corporation, Melbourne, FL, USA) is a novel commercial system that automatically monitors the consistency of daily treatment delivery and calculates the DVH-based QA metrics using the log files and the images from the electronic portal imaging device (EPID). In addition, this system can calculate the threedimensional (3D) patient dose based on forward projection technique not only in the planning CT ( $\mathrm{PCT}$ ) but also in the onboard $\mathrm{kV}$ cone-beam computed tomography (CBCT) acquired for image guidance of position patients receiving radiation therapy. This powerful function aids in detecting interfractional variation of target volume and OARs, and the changes in tumour size and patient weight, through CBCT images for each fraction, and assessment of the potential need for adaptive radiation therapy (ART). Quian et al. demonstrated that dose reconstruction for VMAT using CBCT and log file provided a valuable tool for monitoring the actual dose delivered to the target and OARs [10]. However, the image quality of CBCT is inferior to that of pCT due to the greater noise and poorer contrast that is caused by the increase in radiation scatter in the patient and the wide area detector $[11,12]$.

A new CBCT reconstruction algorithm, iterative CBCT (iCBCT), has recently been introduced into the TrueBeam systems (Varian Medical Systems, Palo Alto, CA, USA). This advanced technique enables efficient scatter collection without additional manual interference, using Acuros
CT scatter and statistical iterative reconstruction. Some researchers determined that $\mathrm{iCBCT}$ increases the contrast-to-noise ratio, maintains Hounsfield unit (HU) uniformity, and reduces streak and metal artifacts and image noise [13-15]. Gardner et al. [14] reported an improved ability to delineate soft tissue structure by using iCBCT images. Washio et al. [16] stated that iCBCT-based dose calculation was more accurate than conventional CBCT-based dose calculation in VMAT for the head and neck. However, none of the previous studies have explored the accuracy of dose calculation using machine log file-based dose reconstruction method in conventional СBCT and iCBCT images. Moreover, a novel online adaptive treatment platform, Ethos (Varian Medical Systems), which is a conversion of Halcyon linear accelerator systems (Varian Medical Systems), has allowed an on-couch plan adaptation by using iCBCT images. The introduction of proton beam therapy, such as FLASH and proton arc therapy, is expected to increase in the future [17], which will further necessitate iCBCT-based dose calculations for ART. It is important that the accuracy of dose calculation in iCBCT images is closely discussed, in order to deal with ART in a general linear accelerator, such as TrueBeam.

This study aims to demonstrate that machine log file-based doses calculated on $\mathrm{iCBCT}$ images are consistent with those calculated on conventionally generated images in a commercial software, during VMAT for prostate cancer patients. This study will contribute to establishing the reliability of dose calculations performed on ICBCT images, thereby expanding the applications of ART. 
(a)
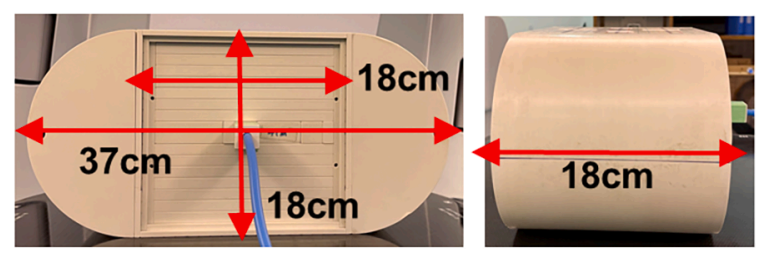

(b)
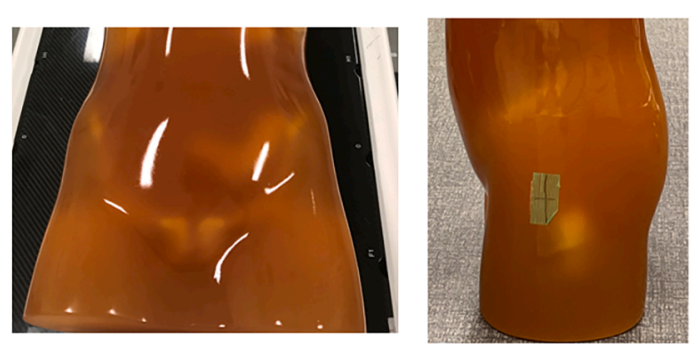

(c)

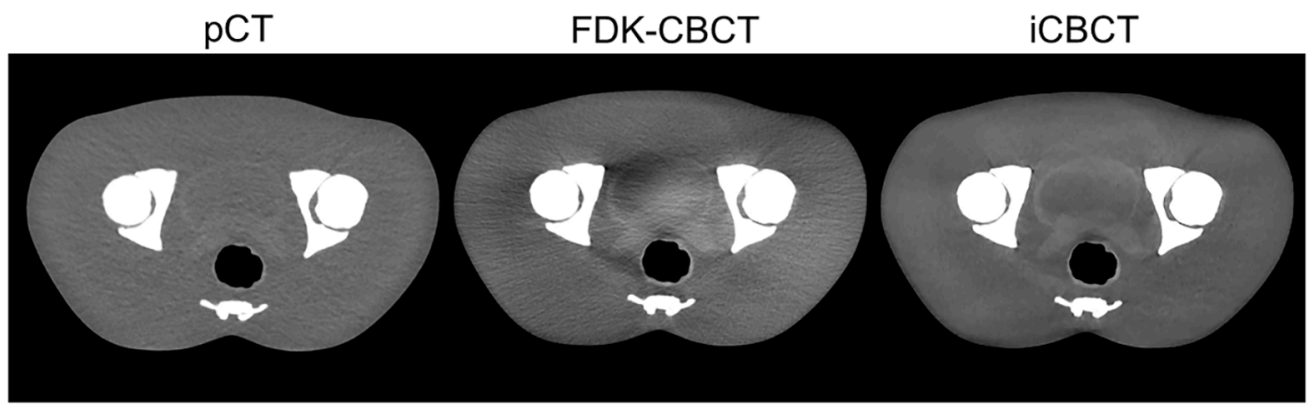

(d)

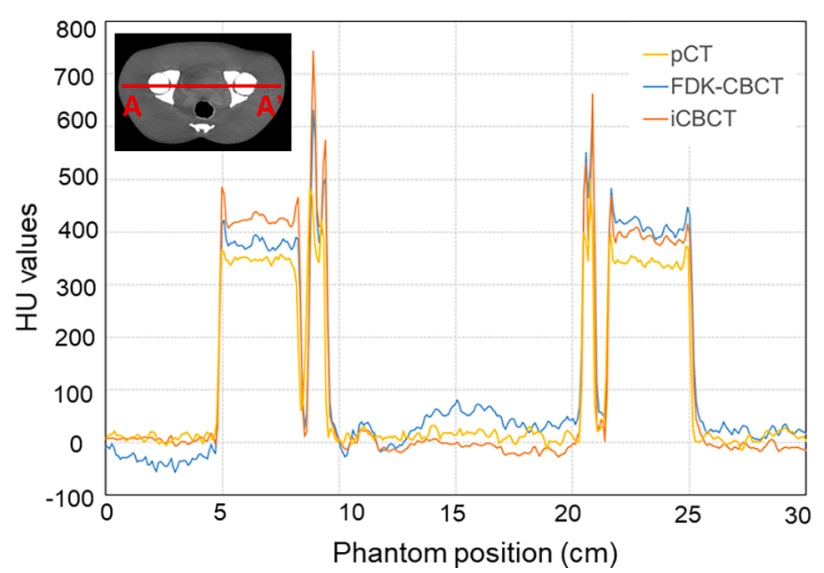

(e)

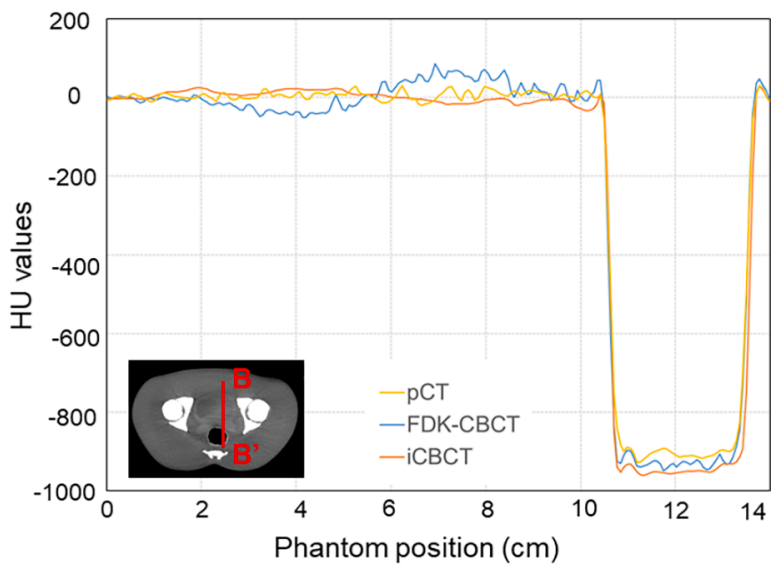

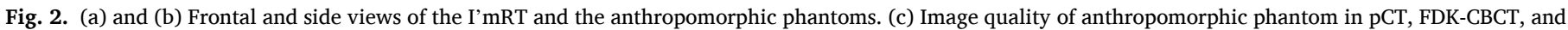
iCBCT images. (d) and (e) Comparison of HU values profiles converted from the AA' and BB' lines on the anthropomorphic phantom.

\section{Methods}

\section{Equipment and CBCT protocol}

All pCT scans were performed using a GE Dual Energy instrument (64 slices, General Electric Co., Waukesha, WI). The parameters of the CT images were $2.0-\mathrm{mm}$ slice thickness and $500-\mathrm{mm}$ field of view (FOV) with dimensions of $512 \times 512$ pixels. All the CT images were transferred to an Eclipse planning system (version 15.6.05, Varian Medical Systems, Palo Alto, CA). In Eclipse, all plans were calculated using the analytical anisotropic algorithm (AAA) for dose calculation with inhomogeneity corrections, and exported to the PerFRACTION software (version 2.2.2, Sun Nuclear Corporation, Melbourne, FL) as DICOM files.

PerFRACTION, which uses GPU-accelerated collapsed cone convolution (CCC) algorithm, named Sun Nuclear Dose Calculator (SDC), and is independent of the Eclipse planning system, is a commercial software for pre- and on-treatment patient-specific QA by using the log file and
EPID images. This system calculates the 3D patient dose using a forward projection technique which incorporates variations in delivered output and MLC positions from planned positions. The output of machine (MU), gantry angle, and treatment parameters were calculated using the log file, and the MLC positions were calculated using either the log file or EPID cine images. In this study, we utilized on-treatment patient-specific QA (Fraction N) mode and performed the 3D patient dose calculation using only the log file. The automatic retrieval of the acquired new log files, and comparison of DVH of a 3D patient dose in pCT and CBCT images is a feature of PerFRACTION. We used the result of pCT calculated using SDC as reference, to eliminate the dose discrepancy between the Eclipse calculation algorithm (AAA) and the SDC algorithm (CCC). The beam model of PerFRACTION was created by the Sun Nuclear Corporation, following the data of percent depth dose, output factor, and profile under a reference condition. The optimization of the beam model was performed by the Sun Nuclear Corporation to minimize the dose error between treatment delivery in our institute and the SDC 
values due to the MLC leaf parameter difference.

All CBCT scans were performed on a linear accelerator, TrueBeam Edge (Varian Medical Systems, Palo Alto, CA, USA) equipped with a gantry-mounted on-board imager (Varian Medical Systems), and taken on the clinical pelvis protocol, consisting of a tube voltage of $125 \mathrm{kVp}$, a tube current time of $1350 \mathrm{mAs}, 2-\mathrm{mm}$ slice thickness, full-arc acquisition, 900 projections, gantry speed of $6 \mathrm{deg} / \mathrm{s}$, and an acquisition time of $60 \mathrm{~s}$. CBCT images acquired using this protocol had an axial FOV diameter of $46.5 \mathrm{~cm}$ and a longitudinal scan length of $17.5 \mathrm{~cm}$. The conventional CBCT removes scatter using a kernel-based correction, followed by filtered back-projection reconstruction, based on the Feldkamp-Davis-Kress algorithm (FDK-CBCT). In this study, FDK-CBCT and iCBCT were reconstructed from the same raw CBCT image for each dataset.

This study is divided into 3 parts. The first includes generation of the HU-electron density (ED) curve for FDK-CBCT and iCBCT using the CBCT calibration phantom. The second involves comparison of plans based on FDK-CBCT and ICBCT in the phantom study. The third involves comparison of 3D patient dose of clinical plans in FDK-CBCT with those in iCBCT. The analysis of the second and third parts was performed by using the PerFRACTION software.

\section{CBCT calibration}

The Advanced Electron Density Phantom (Model 1467, Sun Nuclear Corporation, Melbourne, FL) was used to generate the HU-ED curve for FDK-CBCT and iCBCT, as shown in Fig. 1a. The phantom consisted of inner (diameter $=20 \mathrm{~cm}$ ) and outer $(40 \mathrm{~cm}$ wide and $30 \mathrm{~cm}$ long) sections surrounded by water-equivalent material, which allowed the simulation of the x-ray scatter conditions in the pelvis. This phantom, which contained $2.85 \mathrm{~cm}$ diameter inserts representing the lung lymph node, adipose, breast, brain, liver, and inner bone, was correctly set up on the central axis of TrueBeam Edge, and CBCT scans were performed. Further, CBCT scans were also performed when $10 \mathrm{~cm}$ of the solid water (SW) was added to either end of the phantom to ensure the scatter condition simulated the patient environment. These reconstructed images were transferred to Eclipse for image analysis (Fig. 1b). Circular regions of interest (ROIs) with a 2 -cm diameter were placed at the center of each reference material on FDK-CBCT images. These ROIs were copied and pasted on the iCBCT images to measure mean $\mathrm{HU}$ values and establish HU-ED curves. The HU-ED curves established for FDK-CBCT and $\mathrm{iCBCT}$ were defined as $\mathrm{HU}-\mathrm{ED}_{\mathrm{FDK}}$ and $\mathrm{HU}-\mathrm{ED}_{\mathrm{iCBCT}}$, respectively. The HU-ED $D_{\text {FDK }}$ and HU-ED $D_{\text {iCBCT }}$ curves were set up in PerFRACTION software.

\section{Phantom study}

Two phantom studies were performed in this research. To evaluate 3D dose calculation accuracy in FDK-CBCT and iCBCT images for phantom one, an I'mRT phantom comprising of a body phantom (IBA Dosimetry, Schwarzenbruck, Germany) was used (Fig. 2a). The mock prostate structure set downloaded from AAPM TG-119 was registered on CT images of the I'mRT phantom with MIM Maestro (MIM Software Inc., $\mathrm{OH}$, USA). A single-arc VMAT plan using 2 Gy fractions with 6-MV photons was optimized to deliver the mean of the prescribed dose to the planning target volume (PTV). We performed five CBCT scans of the I'mRT phantom. In the first scan, the I'mRT phantom was setup at the centre of the imaging axis. The remaining four scans offsetting the I'mRT phantom by $\pm 5 \mathrm{~cm}$ in the longitudinal and lateral directions were simulated. This offsetting creates image asymmetry, and the robustness of the reconstruction can be tested. In all the cases, the images were matched in the image guidance, and the irradiation was performed after registration. We acquired the log file and reconstructed CBCT images in all cases. In addition, we measured the dose at the isocenter using a PTW pinpoint ion chamber (Type 31016, PTW, Freiburg, Germany) in each case and compared the measured dose with the reconstructed point dose in each CBCT image of the I'mRT phantom.

For the second phantom study, an anthropomorphic phantom (Kyoto Kagaku, Japan) was used to evaluate the effect of image asymmetry caused by offsetting the patient within the scanner on dose calculation in FDK-CBCT and iCBCT images (Fig. 2b). In CT images of the anthropomorphic phantom, the prostate was contoured as the target volume, while the rectum, bladder, and small and large bowels were contoured as OARs. A single-arc VMAT plan using 2 Gy fractions with 6-MV photons was created for target contouring. The plans were optimized to deliver the mean of the prescribed dose to the PTV. Similar to the first phantom study, we performed five CBCT scans of the anthropomorphic phantom. In all cases, the images were matched in the image guidance, and the irradiation was performed after registration. We acquired the $\log$ files and reconstructed CBCT images in each condition.

\section{Clinical study}

From February 2020 to January 2021, 14 patients with histologically proven low- and intermediate-risk localized prostate cancer underwent VMAT in Osaka International Cancer Institute. The patients' ages lied between 59 and 79 years (median: 74 years). All patients were required to empty their bladder and evacuate their bowels an hour before the CT simulation. For the CT simulation, the patients were immobilized using the Vac-Lock Cushion (Civco Medical Solutions, Iowa, USA) for the pelvis region, while they were also given a leg support and a pillow. Once the immobilization devices were fixed, a pCT scan was performed. If the rectum was deemed too large, the patients had to retake the scan after emptying their rectum. Further, the target volumes and OARs (i.e., the rectum, bladder, and small and large bowels) were contoured by radiation oncologists following the recommendations in Reports 50 and 62 of the International Commission on Radiation Units [18,19]. All the patients were treated with 6-MV photons using single-arc VMAT and received doses of $78 \mathrm{~Gy}$ in 39 fractions. The doses were prescribed at mean dose for the PTV. All the patients received daily soft tissue registration, which was performed using CBCT and pCT in a six degree of freedom couch. If the rectum was full of rectal gas near the prostate, the patient was encouraged to clear their bowels and registration was performed again. We acquired the log files and reconstructed CBCT images in the first treatment session (day 1 ) of all patients.

\section{Data analysis}

We compared DVH metrics calculated by PerFRACTION in FDKCBCT with those in iCBCT images for phantom and clinical studies. For the target, we considered as relevant dose values $\mathrm{D}_{\text {mean }}, \mathrm{D}_{2} \%$, and $\mathrm{D}_{98 \%}$, which represent the dose received by mean, $2 \%$, and $98 \%$ of the PTV. For the bladder and rectum, the volumes that received a specific dose in $35 \mathrm{~Gy}, 50 \mathrm{~Gy}$ and $65 \mathrm{~Gy}\left(\mathrm{~V}_{35 \mathrm{~Gy}}, \mathrm{~V}_{50 \mathrm{~Gy}}\right.$, and $\left.\mathrm{V}_{65 \mathrm{~Gy}}\right)$, and the mean dose were compared. To evaluate dose differences between pCT and each CBCT, the $\% \triangle \mathrm{D}$ was calculated based on the following equation: $\% \Delta \mathrm{D}=\left(\mathrm{D}_{\mathrm{CBCT}}-\mathrm{D}_{\mathrm{pCT}}\right) / \mathrm{D}_{\mathrm{pCT}} \times 100$, where $\mathrm{D}_{\mathrm{CBCT}}$ and $\mathrm{D}_{\mathrm{pCT}}$ were the dose values calculated by using each $\mathrm{CBCT}$ and pCT. The quantitative evaluation of the dosimetric accuracy was performed using the gamma method in 3D patient dose. Gamma analysis criteria of $1 \% / 1 \mathrm{~mm}, 2 \% / 2$ $\mathrm{mm}$, and $3 \% / 3 \mathrm{~mm}$ were used above a $10 \%$ maximum signal threshold, considering the dose distribution calculated by the PCT as a reference. The dose difference among pCT, FDK-CBCT, and iCBCT images was calculated using 3DVH software (Sun Nuclear Corporation). The paired Wilcoxon signed-rank test was performed on the data that did not follow normal distribution (SPSS, version 24; IBM, Armonk, NY), for the statistical measurement of the differences between FDK-CBCT and iCBCT. A $p$-value below 0.05 was considered to indicate statistical significance.

\section{Results}

Fig. 1b shows the comparison between image quality in FDK-CBCT 
Table 1

Measured and calculated iso center dose in I'mRT phantom (the relative difference between the calculated or measured value relative to the pCT-calculated dose).

\begin{tabular}{|c|c|c|c|c|c|}
\hline \multirow[t]{2}{*}{ Method } & \multicolumn{5}{|c|}{ Phantom offset [Gy] } \\
\hline & No offset & $\begin{array}{l}\text { Lng }+5 \\
\text { cm }\end{array}$ & $\begin{array}{l}\text { Lng - } 5 \\
\mathrm{~cm}\end{array}$ & $\begin{array}{l}\text { Lat }+5 \\
\text { cm }\end{array}$ & $\begin{array}{l}\text { Lat - } 5 \\
\text { cm }\end{array}$ \\
\hline pCT & 2.00 & 2.00 & 2.00 & 2.00 & 2.00 \\
\hline FDK-CBCT & $\begin{array}{l}2.02 \\
(+1.10 \%)\end{array}$ & $\begin{array}{l}2.03 \\
(+1.75 \%)\end{array}$ & $\begin{array}{l}2.03 \\
(+1.85 \%)\end{array}$ & $\begin{array}{l}2.01 \\
(+0.65 \%)\end{array}$ & $\begin{array}{l}2.01 \\
(+0.70 \%)\end{array}$ \\
\hline iCBCT & $\begin{array}{l}1.99 \\
(-0.25 \%)\end{array}$ & $\begin{array}{l}2.00 \\
(-0.05 \%)\end{array}$ & $\begin{array}{l}2.00 \\
(+0.00 \%)\end{array}$ & $\begin{array}{l}1.99 \\
(-0.35 \%)\end{array}$ & $\begin{array}{l}1.99 \\
(-0.35 \%)\end{array}$ \\
\hline $\begin{array}{l}\text { Treatment } \\
\text { plan } \\
\text { (calculated } \\
\text { by SDC) }\end{array}$ & $\begin{array}{l}2.00 \\
(-0.10 \%)\end{array}$ & $\begin{array}{l}2.00 \\
(-0.10 \%)\end{array}$ & $\begin{array}{l}2.00 \\
(-0.10 \%)\end{array}$ & $\begin{array}{l}2.00 \\
(-0.10 \%)\end{array}$ & $\begin{array}{l}2.00 \\
(-0.15 \%)\end{array}$ \\
\hline $\begin{array}{l}\text { Measurement } \\
\text { (ion } \\
\text { chamber) }\end{array}$ & $\begin{array}{l}2.01 \\
(+0.75 \%)\end{array}$ & $\begin{array}{l}2.01 \\
(+0.75 \%)\end{array}$ & $\begin{array}{l}2.01 \\
(+0.70 \%)\end{array}$ & $\begin{array}{l}2.01 \\
(+0.50 \%)\end{array}$ & $\begin{array}{l}2.01 \\
(+0.60 \%)\end{array}$ \\
\hline
\end{tabular}

and iCBCT images, in calibration phantom. Compared with FDK-CBCT images, iCBCT images showed a more qualitative reduction in noise. The HU-ED curves for PCT, FDK-CBCT, and iCBCT are shown in Fig. 1c. There were slight differences between the employment and non- employment of SW in HU-ED ${ }_{\mathrm{FDK}}$ and HU-ED ${ }_{\text {iCBCT }}$ curves. The y-intercept value of the linear regression plotting the HU value of the nonemployment of SW in FDK-CBCT was the largest among all, as shown in Fig. 1d.

The summary of measured and calculated isocenter doses at pCT, FDK-CBCT, and iCBCT images obtained using the I'mRT phantom, are shown in Table 1 . Dose differences from pCT were less than $2 \%$ in all cases in a homogenous condition. Table 2 illustrates the differences in HU and ED observed on offset of anthropomorphic phantom scans. The differences in the calculated electron densities under the offset scanning conditions were less than $0.03 \mathrm{~g} / \mathrm{cm}^{3}$. Fig. 2c shows the comparison between image quality in pCT, FDK-CBCT, and iCBCT images in the anthropomorphic phantom. Compared with FDK-CBCT images, iCBCT images also showed a more qualitative reduction in noise. The HU profiles are displayed in Fig. 2d and 2e. The increases and nonuniformity of HU values in FDK-CBCT images were observed at the central position surrounding the bones, in comparison to those in PCT and iCBCT images. Fig. 3 represents the dose distribution among the three different images of the anthropomorphic phantom in axial, coronal, and sagittal views. There were little changes among pCT, FDK-CBCT, and iCBCT in dose distribution. The target dose of iCBCT images was lower than that of FDK-CBCT images. Table 3 shows the dose differences between mean doses to volumes, calculated on each СBCT and pCT in the

Table 2

The mean values of HU and ED when the anthropomorphic phantom is offset within FDK-CBCT and iCBCT scans.

\begin{tabular}{|c|c|c|c|c|c|c|}
\hline \multirow[t]{2}{*}{ Phantom offset } & \multicolumn{3}{|c|}{ HU (FDK-CBCT, iCBCT) } & \multicolumn{3}{|c|}{ ED (FDK-CBCT, iCBCT) [g/cm3] } \\
\hline & Prostate & Bladder & Rectum & Prostate & Bladder & Rectum \\
\hline No offset & $(62.5,25.9)$ & $(5.1,1.8)$ & $(-618.9,-640.8)$ & $(1.03,1.03)$ & $(0.96,1.00)$ & $(0.30,0.33)$ \\
\hline Lng $+5 \mathrm{~cm}$ & $(37.5,7.7)$ & $(-8.1,-14.0)$ & $(-611.4,-632.0)$ & $(1.02,1.00)$ & $(0.95,0.99)$ & $(0.31,0.34)$ \\
\hline Lng $-5 \mathrm{~cm}$ & $(47.7,6.9)$ & $(-9.3,-11.8)$ & $(-627.7,-643.2)$ & $(1.02,1.00)$ & $(0.95,0.99)$ & $(0.29,0.33)$ \\
\hline Lat $+5 \mathrm{~cm}$ & $(54.0,47.4)$ & $(4.9,6.5)$ & $(-611.7,-637.0)$ & $(1.02,1.06)$ & $(0.96,1.00)$ & $(0.31,0.33)$ \\
\hline Lat $-5 \mathrm{~cm}$ & $(64.5,39.6)$ & $(-17.7,18.9)$ & $(-599.1,-639.6)$ & $(1.03,1.05)$ & $(0.94,1.03)$ & $(0.32,0.33)$ \\
\hline
\end{tabular}

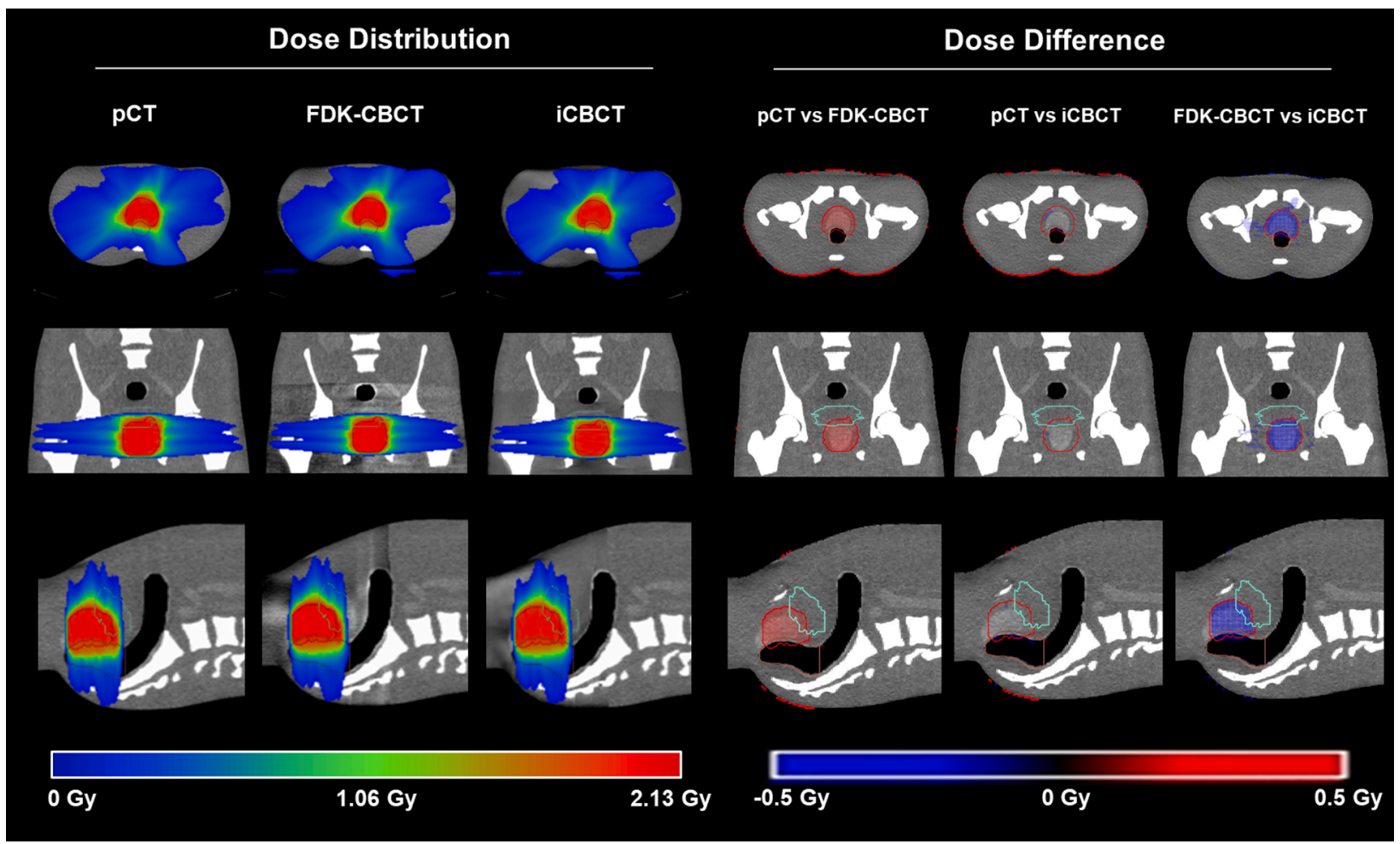

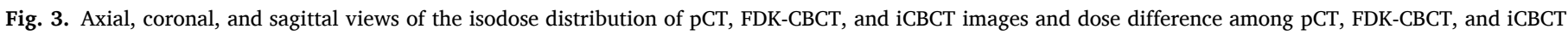

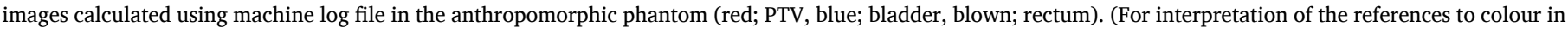
this figure legend, the reader is referred to the web version of this article.) 
Table 3

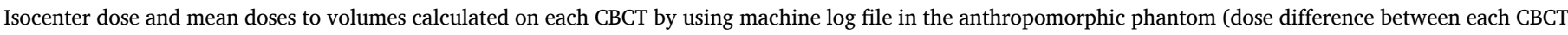
and pCT).

\begin{tabular}{|c|c|c|c|c|c|c|c|c|}
\hline \multirow[t]{2}{*}{ Phantom offset } & \multicolumn{2}{|c|}{ Iso center dose $[\mathrm{Gy}](\%)$} & \multicolumn{2}{|c|}{ PTV D $D_{\text {mean }}[G y](\%)$} & \multicolumn{2}{|c|}{ Bladder $D_{\text {mean }}[G y](\%)$} & \multicolumn{2}{|c|}{ Rectum $D_{\text {mean }}[G y](\%)$} \\
\hline & FDK-CBCT & іСВCT & FDK-CBCT & іСBCT & FDK-CBCT & іСBCT & FDK-СBCT & iCBCT \\
\hline No Offset & $\begin{array}{l}2.05 \\
(1.48)\end{array}$ & $\begin{array}{l}2.01 \\
(-0.94)\end{array}$ & $\begin{array}{l}2.03 \\
(2.79)\end{array}$ & $\begin{array}{l}1.98 \\
(0.41)\end{array}$ & $\begin{array}{l}1.07 \\
(1.91)\end{array}$ & $\begin{array}{l}1.05 \\
(0.00)\end{array}$ & $\begin{array}{l}1.22 \\
(2.79)\end{array}$ & $\begin{array}{l}1.19 \\
(1.02)\end{array}$ \\
\hline $\operatorname{Lng}+5 \mathrm{~cm}$ & $\begin{array}{l}2.06 \\
(1.88)\end{array}$ & $\begin{array}{l}2.02 \\
(-0.35)\end{array}$ & $\begin{array}{l}2.03 \\
(2.84)\end{array}$ & $\begin{array}{l}1.99 \\
(0.76)\end{array}$ & $\begin{array}{l}1.07 \\
(1.91)\end{array}$ & $\begin{array}{l}1.05 \\
(0.19)\end{array}$ & $\begin{array}{l}1.22 \\
(2.96)\end{array}$ & $\begin{array}{l}1.20 \\
(1.27)\end{array}$ \\
\hline Lng $-5 \mathrm{~cm}$ & $\begin{array}{l}2.06 \\
(1.93)\end{array}$ & $\begin{array}{l}2.01 \\
(-0.64)\end{array}$ & $\begin{array}{l}2.03 \\
(2.99)\end{array}$ & $\begin{array}{l}1.99 \\
(0.66)\end{array}$ & $\begin{array}{l}1.07 \\
(2.10)\end{array}$ & $\begin{array}{l}1.05 \\
(0.19)\end{array}$ & $\begin{array}{l}1.22 \\
(3.05)\end{array}$ & $\begin{array}{l}1.20 \\
(1.10)\end{array}$ \\
\hline Lat $+5 \mathrm{~cm}$ & $\begin{array}{l}2.05 \\
(1.48)\end{array}$ & $\begin{array}{l}2.01 \\
(-0.74)\end{array}$ & $\begin{array}{l}2.03 \\
(2.69)\end{array}$ & $\begin{array}{l}1.98 \\
(0.51)\end{array}$ & $\begin{array}{l}1.07 \\
(1.72)\end{array}$ & $\begin{array}{l}1.05 \\
(0.19)\end{array}$ & $\begin{array}{l}1.22 \\
(2.96)\end{array}$ & $\begin{array}{l}1.20 \\
(1.10)\end{array}$ \\
\hline Lat $-5 \mathrm{~cm}$ & $\begin{array}{l}2.06 \\
(1.78)\end{array}$ & $\begin{array}{l}2.01 \\
(-0.89)\end{array}$ & $\begin{array}{l}2.03 \\
(3.04)\end{array}$ & $\begin{array}{l}1.98 \\
(0.51)\end{array}$ & $\begin{array}{l}1.07 \\
(2.20)\end{array}$ & $\begin{array}{l}1.05 \\
(0.19)\end{array}$ & $\begin{array}{l}1.22 \\
(3.05)\end{array}$ & $\begin{array}{l}1.20 \\
(1.27)\end{array}$ \\
\hline
\end{tabular}

Table 4

Results of 3D overall gamma analysis for day 1 FDK-CBCT and iCBCT compared to pCT calculated by using day 1 machine log file.

\begin{tabular}{|c|c|c|c|c|c|c|}
\hline \multirow{2}{*}{$\begin{array}{l}\text { Patient } \\
\text { Number }\end{array}$} & \multicolumn{3}{|c|}{ FDK-CBCT [\%] } & \multicolumn{3}{|c|}{ iCВCT [\%] } \\
\hline & $\begin{array}{l}1 \% / 1 \\
\mathrm{~mm}\end{array}$ & $\begin{array}{l}2 \% / 2 \\
\mathrm{~mm}\end{array}$ & $\begin{array}{l}3 \% / 3 \\
\mathrm{~mm}\end{array}$ & $\begin{array}{l}1 \% / 1 \\
\mathrm{~mm}\end{array}$ & $\begin{array}{l}2 \% / 2 \\
\mathrm{~mm}\end{array}$ & $\begin{array}{l}3 \% / 3 \\
\mathrm{~mm}\end{array}$ \\
\hline 1 & 95.66 & 98.78 & 99.94 & 97.14 & 99.46 & 99.99 \\
\hline 2 & 93.11 & 98.37 & 99.69 & 96.41 & 99.28 & 99.83 \\
\hline 3 & 94.95 & 98.47 & 99.92 & 96.41 & 99.28 & 99.94 \\
\hline 4 & 82.66 & 96.05 & 99.39 & 83.37 & 96.91 & 99.93 \\
\hline 5 & 88.86 & 96.73 & 99.17 & 95.20 & 99.30 & 99.99 \\
\hline 6 & 87.47 & 97.79 & 99.93 & 95.35 & 99.87 & 100 \\
\hline 7 & 96.34 & 99.72 & 99.97 & 93.19 & 99.67 & 99.98 \\
\hline 8 & 94.86 & 98.80 & 99.97 & 92.63 & 98.97 & 99.97 \\
\hline 9 & 87.37 & 98.47 & 99.95 & 95.70 & 99.94 & 100 \\
\hline 10 & 96.53 & 99.19 & 99.97 & 94.64 & 99.13 & 99.96 \\
\hline 11 & 92.27 & 99.01 & 99.89 & 92.52 & 98.93 & 99.73 \\
\hline 12 & 91.59 & 96.67 & 98.62 & 86.39 & 95.52 & 98.55 \\
\hline 13 & 94.51 & 99.10 & 99.97 & 92.42 & 99.49 & 100 \\
\hline 14 & 95.71 & 98.04 & 99.24 & 90.74 & 97.74 & 99.27 \\
\hline
\end{tabular}

anthropomorphic phantom. The dose differences between iCBCT and pCT were lower than those between FDK-CBCT and pCT in all conditions. The dose difference between FDK-СBCT and iCBCT was less than $1 \%$ at each offset. The 3D gamma analysis was performed by using the anthropomorphic phantom calculated on the $\mathrm{pCT}$ and reconstructed CBCT images. The pass rate in FDK-CBCT and iCBCT images was $74.57 \%$ and $82.69 \%$ for $1 \% / 1 \mathrm{~mm}$ analysis, $97.84 \%$ and $99.14 \%$ for $2 \% / 2 \mathrm{~mm}$ analysis, and $99.99 \%$ and $100 \%$ for $3 \% / 3 \mathrm{~mm}$ analysis, respectively.

In the clinical study, the results of the 3D gamma analysis for patients' plans, calculated on PCT and reconstructed CBCT images that were taken on day 1 of treatment, are shown in Table 4 . The average gamma pass rates in FDK-CBCT and iCBCT images were $92.25 \% \pm 4.21$ and $93.06 \% \pm 4.04 \%$ for $1 \% / 1 \mathrm{~mm}$ analysis, $98.22 \% \pm 1.07$ and $98.81 \%$ $\pm 1.25 \%$ for $2 \% / 2 \mathrm{~mm}$ analysis, and $99.68 \% \pm 0.42$ and $99.78 \% \pm$ $0.40 \%$ for $3 \% / 3 \mathrm{~mm}$ analysis, respectively. Fig. 4 shows the differences between the 3D patient dose of PCT and reconstructed CBCT images, in the representative dosimetric parameters of PTV and OARs. The dose differences between iCBCT and pCT were significantly lower than those between FDK-CBCT and PCT in the dosimetric parameters of PTV and OARs.

\section{Discussion}

Previous studies have demonstrated the utility of the CBCT dose calculation for the dosimetric evaluation of changes in the target position and the anatomy, such as tumor shrinkage and patient weight loss, during the treatment $[20,21]$. However, the conventional CBCT (FDKCBCT) images are considered to pose problems related to HU nonuniformity and noise, as opposed to pCT. Novel CBCT (iCBCT) images have been proven to resolve these problems, as compared to FDK-CBCT images [13-15]. Moreover, machine log file-based CBCT calculation is a powerful way to assess the actually delivered 3D patient dose in patientspecific QA [10]. To the best of our knowledge, this is the first study to demonstrate the efficacy of machine log file-based dose verification during VMAT for prostate cancer patients by using the iCBCT image, compared to the FDK-CBCT image in PerFRACTION software.

Sait et al. [22] found that the estimation of point dose calculated by using machine log file in PerFRACTION, is in agreement with measurements using an ionization chamber; similar results were observed in

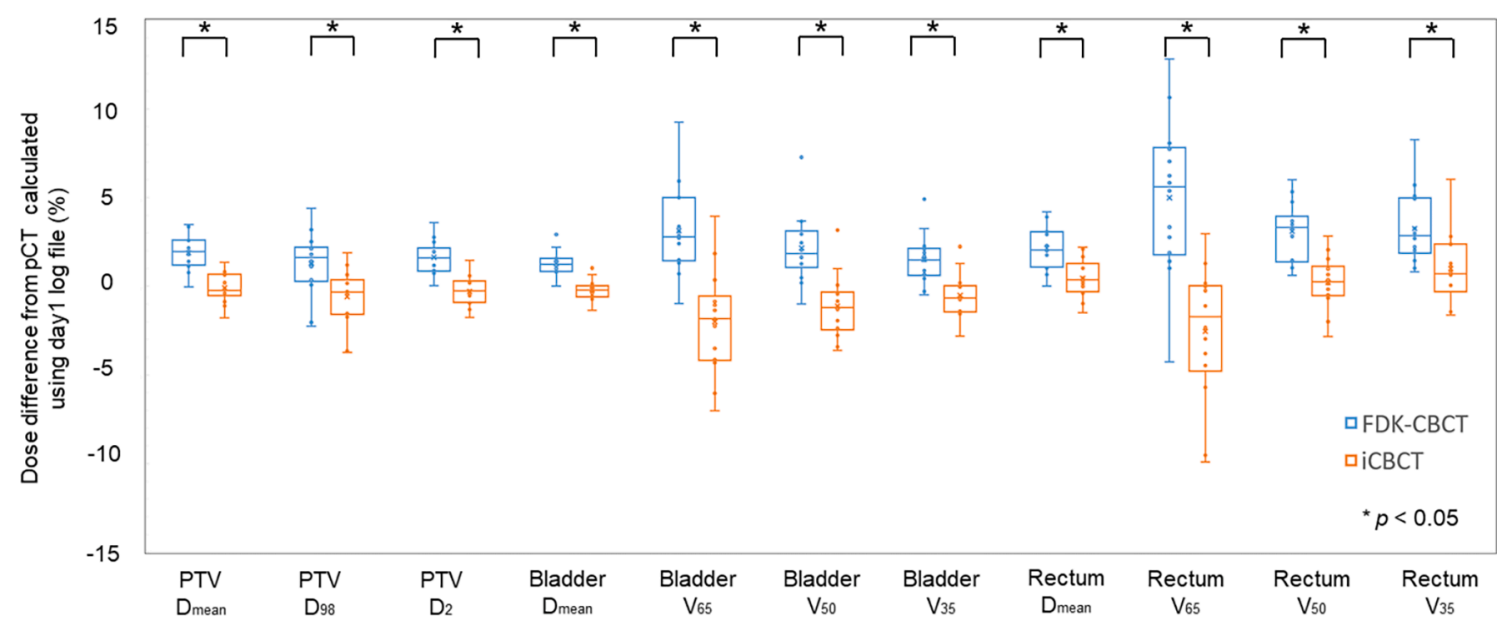

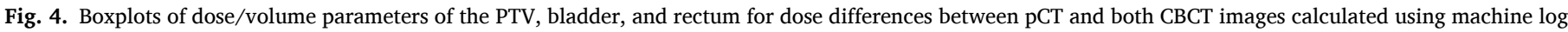
file in clinical study. 
our study. Moreover, we confirmed that there were negligible changes in the isocenter dose among pCT, FDK-СBCT, and iCBCT images in the I'mRT phantom. In addition, we conducted an additional actual dose verification using ArcCHECK (Sun Nuclear Corporation) and an analysis using 3DVH system. The gamma pass rates of $3 \% / 3 \mathrm{~mm}$ and $2 \% / 2 \mathrm{~mm}$ were $99.3 \%$ and $97.8 \%$ between the actual dose measurement and machine log file-based dose reconstruction. Thus, we confirmed almost no difference between the actual dose measurement and machine log file-based dose reconstruction. However, in the anthropomorphic phantom, mean dose difference of PTV and OARs from pCT image in iCBCT image was lower than that in FDK-CBCT image. This may have affected the dose calculation because the HU values near the centre of the target surrounded by pelvic bones were varied with the FDK-CBCT images, but those were almost not varied with the iCBCT images. In addition, offsetting anthropomorphic phantom for FDK-CBCT and iCBCT images shows that electron density and dose calculation do not vary with position in the patient. Jarema et al. [23] reported a similar result in a pelvic phantom study performed using iCBCT images of the Halcyon linear accelerator. Although the anthropomorphic phantom did not shift over $5 \mathrm{~cm}$ due to the limitation of axial FOV diameter and the longitudinal scan length in our study, most of patients have acquired CBCT images at centre position on the imaging axis in order to avoid the collision of patient body and gantry. Subsequently, it is suggested that dose calculation for FDK-СBCT and iCBCT images is not influenced by patient position in the clinical.

To assess the 3D patient dose during VMAT for prostate cancer patients, we measured and analyzed each CBCT taken on day 1 of treatment by using the log file-based dose reconstruction method in the clinical study. Dose differences from pCT of about $2 \%$ in FDK-CBCT image were yielded in $\mathrm{D}_{\text {mean }}$ of PTV and OARs, and approximately similar results were reported in the review of the CBCT-based dose calculation [24]. The dose difference of iCBCT image was significantly smaller than that of FDK-CBCT image. Accordingly, it is clear that the iCBCT yields dose distribution markers that are more consistent with those calculated using the PCT and TPS in the log file-based dose verification during VMAT for prostate cancer patients. Further, the $\mathrm{V}_{65 \mathrm{~Gy}}$ of the bladder and rectum exhibited a larger variation than the corresponding $\mathrm{V}_{35 \mathrm{~Gy}}$ and $\mathrm{V}_{50 \mathrm{~Gy}}$ for each OAR. In addition, there were small variations for both CBCT images in a clinical sense because it was only with the strictest threshold criteria of $1 \% / 1 \mathrm{~mm}$ that showed gamma passing rated below $95 \%$. This can be attributed to patient-specific changes between the conditions that CBCT and pCT in target and OARs contours was performed under, such as the situation of the bladder and rectum. Several studies have reported the difficulty in completely controlling bladder and rectum filling, even if patients follow the bladder and rectum preparation protocol prior to treatment $[25,26]$. To achieve more accurate dose calculations using CBCT, deformable image registration (DIR) should be performed in target and OARs' contours.

Previous studies reported that 2D EPID-based in vivo dosimetry can detect rectum and bladder fillings during VMAT for prostate cancer patients $[27,28]$; however, this method is unable to show 3D dosimetric changes due to rectum and bladder fillings. Accordingly, it is important to assess the dose actually delivered to the target as well as OARs by using machine log file-based CBCT calculation. In terms of the accuracy of MLC position, the machine log file-based dose reconstruction method cannot always identify systematic machine faults. Neal et al. [29] reported that machine log file-derived MLC positions can differ from their actual position by $>1 \mathrm{~mm}$. This may be because the machine log file could not record small MLC position errors caused by linac component miscalibration and broken t-nuts. Kadoya et al. [30] reported that log file with cine EPID image may detect these MLC position errors though image acquisition failure and noise are included in PerFRACTION. Further investigation should be conducted to verify whether cine-based MLC positions are likely to further improve the accuracy of MLC log filebased calculations.

This study comes with its own set of limitations. First, our results based on the HU-ED curve should only apply to large thick parts, such as the pelvic region, and not to thin parts, such as the head and neck. In general, the human pelvis is larger than other sites, which results in larger scatter fraction. Rong et al. [31] reported that the HU uniformity deterioration increases according to the size of the scanned objects, mostly due to scatter contamination. Therefore, large differences in body thickness may lead to different results. Second, our data may include uncertain values due to various patient sizes. The difference in size of patient produces the difference in scattering occurrence. Third, there are differences between PCT and СВCT images taken on day 1 , such as random patient setup error and anatomical changes. We also did not consider DIR in this study. However, despite these limitations, our quantitative data makes a significant contribution to the field of radiation therapy with regards to ART.

\section{Conclusion}

The accuracy of machine log file-based dose verifications of iCBCT images was consistent with that of conventionally generated images in a commercial software during VMAT for prostate cancer patients. Based on the results of this study, machine log file-based dose calculation using iCBCT images can expand the application of ART.

\section{Declaration of Competing Interest}

The authors declare that they have no known competing financial interests or personal relationships that could have appeared to influence the work reported in this paper.

\section{Acknowledgements}

We would like to thank Editage (www.editage.jp) for English language editing. This study was supported by JSPS KAKENHI Grant (Grant-in-Aid for Young Scientists 20K16843).

\section{References}

[1] Mehrens H, Taylor P, Followill DS, Kry SF. Survey results of 3D-CRT and IMRT quality assurance practice. J Appl Clin Med Phys. 2020;21:70-6. https://doi.org/ $10.1002 / \mathrm{acm} 2.12885$.

[2] Miften M, Olch A, Mihailidis D, Moran J, Pawlicki T, Molineu A, Li H, Wijesooriya K, Shi J, Xia P, Papanikolaou N, Low DA. Tolerance limits and methodologies for IMRT measurement-based verification QA: Recommendations of AAPM Task Group No. 218. Med Phys. 2018;45:e53-e83. https://doi.org/10.1002/mp.12810.

[3] Kruse JJ. On the insensitivity of single field planar dosimetry to IMRT inaccuracies. Med Phys. 2010;37:2516-24. https://doi.org/10.1118/1.3425781.

[4] Zhen H, Nelms BE, Tome WA. Moving from gamma passing rates to patient DVHbased QA metrics in pretreatment dose QA. Med Phys. 2011;38:5477-89. https:// doi.org/10.1118/1.3633904.

[5] Nelms BE, Zhen H, Tome WA. Per-beam, planar IMRT QA passing rates do not predict clinically relevant patient dose errors. Med Phys. 2011;38:1037. https:// doi.org/10.1118/1.3544657.

[6] Carrasco P, Jornet N, Latorre A, Eudaldo T, Ruiz A, Ribas M. 3D DVH-based metric analysis versus per-beam planar analysis in IMRT pretreatment verification. Med Phys. 2012;39:5040-9. https://doi.org/10.1118/1.4736949.

[7] Tyagi N, Yang K, Yan D. Comparing measurement-derived (3DVH) and machine log file-derived dose reconstruction methods for VMAT QA in patient geometries. J Appl Clin Med Phys 2014;15:4645. https://doi.org/10.1120/jacmp.v15i4.4645.

[8] Sun B, Rangaraj D, Boddu S, Goddu M, Yang D, Palaniswaamy G, et al. Evaluation of the efficiency and effectiveness of independent dose calculation followed by machine log file analysis against conventional measurement based IMRT QA. J Appl Clin Med Phys. 2012;13:3837. https://doi.org/10.1120/jacmp.v13i5.3837.

[9] Calvo-Ortega JF, Teke T, Moragues S, Pozo M, Casals-Farran J. A Varian DynaLog file-based procedure for patient dose-volume histogram-based IMRT QA. J Appl Clin Med Phys. 2014;15:4665. https://doi.org/ 10.1120/jacmp.v15i2.4665.

[10] Qian J, Lee L, Liu W, Chu K, Mok E, Luxton G, et al. Dose reconstruction for volumetric modulated arc therapy (VMAT) using cone-beam CT and dynamic log files. Phys Med Biol. 2010;55:3597-610. https://doi.org/10.1088/0031-9155/55/ $13 / 002$.

[11] Richter A, Hu Q, Steglich D, Baier K, Wilbert J, Guckenberger M, et al. Investigation of the usability of conebeam CT data sets for dose calculation. Radiat Oncol. 2008;3:42. https://doi.org/10.1186/1748-717X-3-42.

[12] Yang Y, Schreibmann E, Li T, Wang C, Xing L. Evaluation of on-board kV cone beam CT (CBCT)-based dose calculation. Phys Med Biol. 2007;52:685-705. https://doi.org/10.1088/0031-9155/52/3/011. 
[13] Mao W, Liu C, Gardner SJ, Siddiqui F, Snyder KC, Kumarasiri A, Zhao B, Kim J, Wen NW, Movsas B, Chetty IJ. Evaluation and Clinical Application of a Commercially Available Iterative Reconstruction Algorithm for CBCT-Based IGRT. Technol Cancer Res Treat. 2019;18:1533033818823054. https://doi.org/10.1177/ 1533033818823054.

[14] Gardner SJ, Mao W, Liu C, Aref I, Elshaikh M, Lee JK, et al. Improvements in CBCT image quality using a novel iterative reconstruction algorithm: a clinical evaluation. Adv Radiat Oncol. 2019;4:390-400. https://doi.org/10.1016/j. adro.2018.12.003.

[15] Washio H, Ohira S, Funama Y, Morimoto M, Wada K, Yagi M, et al. Metal artifact reduction using iterative CBCT reconstruction algorithm for head and neck radiation therapy: A phantom and clinical study. Eur J Radiol. 2020;132:109293. https://doi.org/10.1016/j.ejrad.2020.109293.

[16] Washio H, Ohira S, Funama Y, Ueda Y, Isono M, Inui S, et al. Accuracy of dose calculation on iterative CBCT for head and neck radiotherapy. Phys Med. 2021;86 106-12. https://doi.org/10.1016/j.ejmp.2021.05.027.

[17] Czerska K, Emert F, Kopec R, Langen K, McClelland JR, Meijers A, Miyamoto N, Riboldi M, Shimizu S, Terunuma T, Zou W, Knopf A, Rucinski A. Clinical practice vs. state-of-the-art research and future visions: Report on the 4D treatment planning workshop for particle therapy - Edition 2018 and 2019. Phys Med. 2021; 82:54-63. https://doi.org/10.1016/j.ejmp.2020.12.013.

[18] ICRU (International Commission on Radiation Units and Measurements). Prescribing, recording, and reporting photon beam therapy. ICRU Report 50. ICRU, Washington 1993: 27-8.

[19] ICRU (International Commission on Radiation Units and Measurements). Prescribing, recording, and reporting photon beam therapy (Supplement to ICRU Report 50). ICRU Report 62. ICRU, Bethesda 1999: 3-11.

[20] Ding GX, Duggan DM, Coffey CW. Accurate patient dosimetry of kilovoltage conebeam CT in radiation therapy. Med Phys. 2008;35:1135-44. https://doi.org/ 10.1118/1.2839096.

[21] Jaffray DA, Siewerdsen JH, Wong JW, Martinez AA. Flat-panel cone-beam computed tomography for image-guided radiation therapy. Int J Radiat Oncol Biol Phys. 2002;53:1337-49. https://doi.org/10.1016/s0360-3016(02)02884-5.

[22] Sait AA, Figueredo J, Jones GW, Jones K, McGowan T, Kapatoes J, et al. Validation of three-dimensional electronic portal imaging device-based PerFRACTION ${ }^{\mathrm{TM}}$ software for patient-specific quality assurance. J Med Phys. 2019;44:16-20. https://doi.org/10.4103/jmp.JMP_76_18.

[23] Jarema T, Aland T. Using the iterative kV CBCT reconstruction on the Varian Halcyon linear accelerator for radiation therapy planning for pelvis patients. Phys Med. 2019;68:112-6. https://doi.org/10.1016/j.ejmp.2019.11.015.

[24] Giacometti V, Hounsell AR, McGarry CK. A review of dose calculation approaches with cone beam CT in photon and proton therapy. Phys Med. 2020;76:243-76. https://doi.org/10.1016/j.ejmp.2020.06.017.

[25] McNair HA, Wedlake L, Lips IM, Andreyev J, Van Vulpen M, Dearnaley D. A systematic review: effectiveness of rectal emptying preparation in prostate cancer patients. Pract Radiat Oncol. 2014;4:437-47. https://doi.org/10.1016/j. prro.2014.06.005.

[26] O'Doherty UM, McNair HA, Norman AR, Miles E, Hooper S, Davies M, et al. Variability of bladder filling in patients receiving radical radiotherapy to the prostate. Radiother Oncol. 2006;79:335-40. https://doi.org/10.1016/j. radonc.2006.05.007.

[27] Inui S, Ueda Y, Ono S, Ohira S, Isono M, Nitta Y, et al. Evaluation of twodimensional electronic portal imaging device using integrated images during volumetric modulated arc therapy for prostate cancer. Rep Pract Oncol Radiother. 2021;26:281-90. https://doi.org/10.5603/rpor.a2021.0041.

[28] Fiagan YAC, Bossuyt E, Nevens D, Dirix P, Theys F, Gevaert T, et al. In vivo dosimetry for patients with prostate cancer to assess possible impact of bladder and rectum preparation. Tech Innov Patient Support Radiat Oncol. 2020;16:65-9. https://doi.org/10.1016/j.tipsro.2020.10.005.

[29] Neal B, Ahmed M, Kathuria K, Watkins T, Wijesooriya K, Siebers J. A clinically observed discrepancy between image-based and log-based MLC positions. Med Phys. 2016;43(6):2933-5. https://doi.org/10.1118/1.4949002.

[30] Kadoya N, Kon Y, Takayama Y, Matsumoto T, Hayashi N, Katsuta Y, et al. Quantifying the performance of two different types of commercial software programs for 3D patient dose reconstruction for prostate cancer patients: Machine log files vs. machine log files with EPID images. Phys Med. 2018;45:170-6. https:// doi.org/10.1016/j.ejmp.2017.12.018.

[31] Rong Y, Smilowitz J, Tewatia D, Tomé WA, Paliwal B. Dose Calculation on KV Cone Beam CT Images: An Investigation of the Hu-Density Conversion Stability and Dose Accuracy Using the Site-Specific Calibration. Med Dosim. 2010;35:195-207. https://doi.org/10.1016/j.meddos.2009.06.001. 